\title{
La Covid 19. Un campo de experimentación para el enfoque criminológico de la oportunidad delictiva
}

La criminología es una ciencia empírica, que basa su conocimiento en el análisis de la realidad mediante el método inductivo. No obstante, la naturaleza de su objeto de estudio, -el crimen-, dificulta en gran medida la utilización, por parte de los investigadores del método experimental, consistente en la observación de los hechos no como se presentan de por sí, sino provocados o manipulados intencionalmente para poder establecer las distintas variables que afectan a un determinado objeto de estudio. En la medida en que la metodología experimental se vale de manipulaciones (generalmente de una variable) y de test controlados para poder determinar procesos causales, puede ser éticamente incompatible con gran parte de las investigaciones sobre el fenómeno criminal. Por una parte, no es éticamente viable provocar el crimen para posibilitar su estudio; y, por otra, con la excepción de los denominados casos terapéuticos en los que se mejora o neutraliza alguna variable de sujetos en situación de riesgo delictivo para comprobar el efecto causal de la misma en el comportamiento criminal, es muy difícil poder manipular, a efectos de observación experimental, la mayoría de las variables que pueden influir en el fenómeno delictivo.

La situación derivada de la Covid 19, en concreto el periodo de confinamiento y las limitaciones de los locales y espacios de ocio, así como de los eventos públicos (fiestas, futbol, cine, teatro, etc.) ha configurado el escenario perfecto para un análisis experimental de las teorías criminológicas basadas en el enfoque de la oportunidad delictiva, cuya hipótesis es que la confluencia de determinados elementos en un mismo espacio y tiempo facilitan la comisión del delito.

En la actualidad, la mayor parte de la doctrina criminológica parte de la premisa de que el crimen es un producto de la interacción del individuo con el ambiente en el que se desarrolla; esto es, de la acción recíproca entre factores personales, sociales y situacionales o físicos. Y ello, con independencia de que, según la tesis planteada, se otorgue una mayor importancia a alguno de estos factores frente a los otros.

En este contexto, las teorías situacionales van más allá del estudio de la inclinación delictiva del sujeto, al incluir en el análisis del suceso criminal las características del contexto situacional y/o ambiental que ayuda a que el sujeto con inclinaciones delictivas pase a la acción. Cada son más los trabajos criminológicos que evidencian como determinados escenarios físicos proporcionan muchas más oportunidades delictivas que otros (Felson y Clarke, 1998).

Cómo citar este trabajo: RODRÍGUEZ MESA, María José, "La Covid 19. Un campo de experimentación para el enfoque criminológico de la oportunidad delictiva", Revista de Estudios Jurídicos y Criminológicos, n. ${ }^{\circ}$ 4, Universidad de Cádiz, 2021, pp. 15-21, DOI: https://doi.org/10.25267/REJUCRIM.2021.i.4.02 
Entre las teorías situacionales, la teoría de las actividades rutinarias -también conocida como teoría de la oportunidad- formulada por Cohen y Felson (1979), relaciona el comportamiento delictivo con el contexto situacional del autor y de la víctima a partir de la convergencia en el espacio y en el tiempo de tres elementos básicos: un posible delincuente, un objetivo apropiado y la ausencia de un vigilante adecuado.

También entre las corrientes situacionales o ambientalistas, la teoría del patrón delictivo formulada por Brantingham y Brantingham (1991) parte del análisis del movimiento en el tiempo y en el espacio de los sujetos y objetos involucrados en el hecho criminal. Para ello se tienen en cuenta tres elementos claves: los "Nodos" (desde y hacia donde se trasladan los individuos); las "Rutas" (itinerarios seguidos por las personas en sus actividades cotidianas): y los "Límites" (lindes de las áreas donde la gente vive y lleva a cabo sus actividades diarias).

Tanto una como otra parten de la hipótesis de que las oportunidades desempeñan un papel importante en la comisión del delito, de modo que la reducción de oportunidades delictivas puede producir un descenso del delito. Al depender de los movimientos cotidianos y estar concentradas en el tiempo y en el espacio, la alteración de cualquiera de estos elementos tendría una repercusión en las oportunidades para delinquir, provocando un incremento o descenso de la actividad delictiva. Aunque según esta teoría la reducción de las oportunidades delictivas no suele desplazar el delito, lo cierto es que varios estudios empíricos han puesto de manifiesto que la reducción de la oportunidad mediante el incremento de vigilancia en determinadas zonas provoca un desplazamiento de los delitos patrimoniales a lugares con menor vigilancia. Por otra parte, el incremento del uso cotidiano de las TIC, junto con el hecho de que el ciberespacio sea un escenario en el que no existen distancias físicas ni guardianes formales institucionalizados, favorece el desplazamiento del cibercriminal hacia otros objetivos de ataque (Miró Llinares, 2011).

Pues bien, el cambio sin precedentes que ha supuesto la Covid 19 en las actividades cotidianas de los individuos supone un campo de experimentación natural en el que comprobar algunas de las hipótesis de las teorías de la oportunidad. El periodo de confinamiento tuvo dos consecuencias importantes a estos efectos: por una parte, los individuos estaban recluidos en sus casas, lo que reducía de forma drástica las oportunidades de que el sujeto motivado para delinquir coincidiera con una víctima idónea en el espacio y en el tiempo. Por otra, los individuos modificaron muchas de sus actividades cotidianas, utilizando el ciberespacio como lugar al que se trasladaron gran parte de las actividades que anteriormente se llevaban a cabo en el espacio físico (teletrabajo, compras, ocio, etc.). Tras el periodo de confinamiento estricto, es preciso analizar también si las restricciones de los lugares de ocio, la reducción del turismo, la instauración del teletrabajo y el mantenimiento de actividades en el ciberespacio por parte de los nuevos usuarios han tenido alguna repercusión en la actividad delictiva.

A partir del análisis de los datos sobre criminalidad de 2020 y su comparación con los de 2019 puede comprobarse si, en efecto, el cambio en las actividades cotidianas ha supuesto una reducción de los delitos o un mero desplazamiento de los mismos. También es importante verificar si la reducción de oportunidades para delinquir ha afectado de la 
misma forma a todos los tipos de delincuencia. Para ello se va a partir del dato de los hechos delictivos conocidos por la policía publicados en el Anuario del Ministerio del Interior de 2020, la evolución trimestral de la criminalidad (Ministerio del Interior) y el Estudio sobre la cibercriminalidad en España en 2020 (Ministerio del Interior).

Para cuantificar y comparar las tasas de criminalidad vamos a utilizar el dato de hechos conocidos por la policía (infracciones penales conocidas por los cuerpos policiales en el lugar de ocurrencia del hecho). La tasa de criminalidad, al indicarnos el número de infracciones por cada 1.000 habitantes, permite comparar tipologías delictivas, distintos periodos y países sin que las variaciones en la población afecten a la tasa.

A partir del año 2009 comienza un descenso paulatino de las tasas de criminalidad en nuestro país, de modo que en el año 2017 la tasa de criminalidad era inferior en casi ocho puntos a la de 2009. En los 2018 y 2019 se produce un ligero repunte de la criminalidad en uno y dos puntos respectivamente. El año 2020 cierra con la menor tasa de criminalidad conocida desde que se viene computando en nuestro país, concretamente 37,2 infracciones conocidas por cada 1.000 habitantes, lo que supone un descenso con respecto al año anterior de casi nueve puntos.

Es evidente, y así se reconoce por el propio Ministerio del Interior, que la pandemia ha desempeñado un papel crucial en este acentuado descenso de la criminalidad. Las cuestiones a analizar son: a) si ello es consecuencia de un cambio en las actividades cotidianas con la consecuente reducción de las oportunidades para delinquir; b) si el descenso ha sido generalizado en todas las tipologías delictivas; c) si se ha producido un desplazamiento de la actividad criminal a los nuevos ámbitos de la actividad cotidiana; y d) si se trata de un fenómeno puntual o es posible que perdure más allá de los periodos de restricciones más estrictos.

a) El periodo de confinamiento en España comprendió del 15 de marzo al 21 de junio de 2020, aunque a partir del 10 de mayo comenzó la fase de desescalada, con una progresiva reducción de las restricciones. Aunque los dos primeros meses del año 2020 comenzaron con un incremento de las tasas de criminalidad, durante el periodo de confinamiento según los datos oficiales- la criminalidad (hechos conocidos) se redujo respecto del año anterior en un $42 \%$ en el mes de marzo, $65,5 \%$ en el mes de abril y $40 \%$ en el mes de mayo. Si bien en porcentajes no tan altos, la criminalidad siguió siendo inferior en los meses siguientes, siendo de destacar el mes de diciembre, en el que el porcentaje se redujo respecto al año anterior en un $20 \%$.

El hecho de que la mayor reducción de la criminalidad se corresponda con el periodo en el que durante todo el mes se decretó un confinamiento estricto (abril) es una evidencia de que el cambio en las actividades cotidianas de la población incide en las oportunidades delictivas, reduciéndolas o incrementándolas. El confinamiento evitó en gran medida que el delincuente motivado y la potencial víctima coincidieran en el espacio y en el tiempo físico, lo que conforme a la hipótesis de la teoría de las actividades rutinarias ha de llevar a una reducción del delito. Por otra parte, las restricciones en materia de ocio y de 
reuniones familiares explicaría el descenso de la criminalidad en los meses de julio, agosto y diciembre.

b) Aunque el descenso de la tasa de criminalidad ha sido generalizado a partir de mediados de marzo, no todas las categorías delictivas han experimentado una reducción similar. Entre los delitos contra las personas, el descenso más acusado se ha producido en los delitos de lesiones y riña tumultuaria, con una reducción del $20 \%$ con respecto al año 2019, seguido por los delitos contra la libertad e indemnidad sexual que experimentaron una disminución del 13,6\%. Con respecto a las lesiones y riñas tumultuarias, se trata de delitos muy vinculados a los lugares de ocio y a la interacción entre agresores y víctimas; mientras que los delitos contra la libertad sexual de adultos se cometen principalmente en el domicilio del agresor (63\%) y en portales, parques y vehículos (34\%), lo que implica también una presencia de la potencial víctima en la vía pública o su traslado al domicilio del agresor. Tanto en un caso como en otro, es evidente que la restricción de movimientos y del ocio han influido causalmente en la reducción de este tipo de delincuencia, pues el cambio en las actividades cotidianas ha reducido las oportunidades delictivas.

No obstante, la categoría delictiva con mayor variación con respecto al año anterior ha sido la relativa a los delitos contra el patrimonio, y especialmente los hurtos, que han disminuido en un $40 \%$, seguidos por los robos con violencia e intimidación con una reducción del $31 \%$, y los robos con fuerza en domicilio, establecimientos y otras instalaciones, disminuidos en un 27,5\%. A pesar de la disminución de los robos con violencia o intimidación, es de destacar un incremento de los robos con violencia en vía pública $(16 \%)$ y en establecimientos $(9,4 \%)$. Este último dato, puesto en relación con la disminución de los hurtos y de los robos con fuerza, no niega necesariamente la hipótesis principal de las teorías de la oportunidad, pues es posible que la reducción del turismo, del ocio, y en general de las aglomeraciones de personas hayan reducido las oportunidades para el hurto y robo con fuerza, configurándose la violencia como un instrumento necesario para la comisión del delito ante el cambio en las actividades rutinarias. No obstante, se trata de una cuestión cuyo análisis requiere más atención de lo que se le puede prestar en un trabajo de esta naturaleza.

La categoría delictiva que ha sufrido un incremento histórico ha sido la de los delitos contra el orden público, que se han visto incrementados en un $38 \%$, como consecuencia de las denuncias por atentado contra miembros de las fuerzas armadas y resistencia y desobediencia grave a la autoridad a causa de las manifestaciones en contra del estado de alarma, así como las denuncias por la desobediencia de las restricciones establecidas y del toque de queda. En el ámbito de la LO 4/2015 de Protección a la Seguridad Ciudadana, durante los meses de marzo y abril de 2020 el número de sanciones se incrementaron en un $9.690 \%$ y el $2.138 \%$ respectivamente con relación a los mismos meses del año anterior.

También se han incrementado las estafas en un 10\%, especialmente las relacionadas con cuestiones relativas a la pandemia (estafas por teléfono, datos personales, estafas bancarias, etc.). 
Otra categoría delictiva que ha sufrido un incremento ha sido el tráfico de drogas, que experimentado un aumento del 2,2 \% respecto del año anterior. En el caso del "menudeo", al tratarse de un delito de confluencia en el que es preciso un sujeto dispuesto a vender droga y otro dispuesto a comprarla, el cambio en las actividades cotidianas no les afecta del mismo modo que a los delitos vistos anteriormente, ya que el hecho delictivo no depende en este caso tanto de la oportunidad como de la demanda. En cuanto al narcotráfico, al no existir una víctima directa, su comisión no exige la confluencia en el espacio-tiempo de la que parte la teoría de la oportunidad, por lo que ni el confinamiento ni las restricciones del ocio reducen las oportunidades delictivas para esta categoría delictiva.

Contra todo pronóstico, donde se han reducido los casos de criminalidad -al menos oficial- ha sido en el ámbito de la violencia de género. No obstante, son varias las causas que pueden explicar esta reducción. En primer lugar, la disminución no ha sido tan relevante, ya que en el año 2019 el número de victimizaciones experimentó el mayor incremento histórico, pasando de 77.562 casos en 2016 a 90.674 casos en 2019, por lo que el descenso en 2020 de 3.200 casos sigue manteniendo unas cifras de victimización muy superiores a las de los años precedentes a 2019. Además, hay que tener en cuenta otros dos datos: por una parte, que gran parte de la violencia de género se da por parte de exparejas que, durante el confinamiento, no pudieron acercarse a sus víctimas. Precisamente este dato sirve para explicar el descenso de mujeres muertas con respecto al anterior año en un 19\%; y, por otra, que durante el confinamiento no era el mejor momento para interponer una denuncia por violencia de género ante la incertidumbre de lo que podía pasar con la mujer y los hijos, en su caso, tras poner la denuncia.

En cuanto al número de victimizaciones por violencia familiar el descenso ha sido solo del $0,8 \%$, y al igual que en el caso de la violencia de género sigue representando cifras muy altas respecto a los años anteriores a 2019.

c) Uno de los postulados de la teoría de la oportunidad es que la reducción de las oportunidades delictivas no tiene por qué significar un desplazamiento del delito. Los cambios en la actividad cotidiana durante la pandemia han demostrado, en efecto, que la reducción de oportunidades delictivas ha supuesto una importante disminución global de la criminalidad. Pero también es cierto, que parte de la delincuencia se ha trasladado de unas categorías delictivas a otras, como ha ocurrido con las estafas o con el tráfico de drogas; y, sobre todo, se ha desplazado en el espacio. No de un espacio físico a otro, sino del espacio físico al ciberespacio.

Tanto el traslado de gran parte de la actividad cotidiana (trabajo, ocio, compras, reuniones de amigos, etc.) al ciberespacio, como el incremento del número de usuarios conectados en la red han creado el escenario idóneo para la cibercriminalidad; esto es, para el crimen cometido en el ciberespacio que, como afirma Miró Llinares (2011), se configura como un nuevo ámbito de oportunidad delictiva en el que el tiempo y el espacio se contraen, pero a la vez se expanden, de modo que mientras que en el espacio físico es preciso que los sujetos coincidan en el mismo tiempo y lugar para poder comunicarse, en el 
ciberespacio pueden comunicarse al mismo tiempo o en tiempo separados, y en el mismo ciberespacio pero en espacios físicos distintos.

Si se sigue la hipótesis principal de la teoría de las actividades rutinarias, el incremento de personas y objetos en el ciberespacio junto con la ausencia de controles efectivos para evitar el delito, supondría la existencia de un ámbito en el que las oportunidades delictivas se incrementan de manera exponencial. Si a ello le unimos la reducción de oportunidades delictivas en el espacio físico que ha supuesto la Covid 19, es de esperar que los ciberdelitos hayan experimentado un notable incremento en el año 2020, al coincidir en ese nuevo espacio sujetos motivados para delinquir con víctimas y objetos atractivos.

Según el estudio sobre la cibercriminalidad en España (2020), el total de hechos delictivos conocidos cometidos en el ciberespacio se incrementó en el año 2020 en un 131,9\% frente al año 2019. Según los datos proporcionados por el Sistema Estadístico de Criminalidad, en el año 2020 el 16,3\% de las infracciones penales fueron cometidas en el ciberespacio, frente al 9,9\% del año anterior. Las victimizaciones, por su parte, suponen un 29,7\% más que en el año 2019 y por el perfil de la víctima (varón de entre 26 y 40 años) está muy relacionada con el teletrabajo.

De entre todas las categorías delictivas la sufrió un mayor incremento fue la de fraude informático (89,6\% de los ciberdelitos conocidos), que aumentó respecto al año anterior en un 134\%. Además, los ciberdelincuentes han aprovechado la pandemia para lanzar ataques de ransomware contra las infraestructuras esenciales e instituciones sanitarias necesarias para la lucha contra la pandemia. A nivel europeo, y no solo nacional, se ha registrado un incremento de las campañas de phishing o estafas, que han llegado a constituir el $59 \%$ de las ciberamenazas.

Aunque según los datos del Ministerio del Interior los delitos sexuales se han mantenido sin cambio en el ciberespacio, es preciso tener en cuenta que estamos ante una categoría delictiva en la que existe una alta cifra negra de criminalidad debido a la dificultad de su detección -incluso por parte de la víctima- y de la ausencia de denuncias. Así, según la información facilitada por el Departamento de delitos telemáticos de la Guardia Civil, los reportes sobre supuesta distribución de material pornográfico ilícito se incrementaron en España en un $449 \%$ diario, alcanzando un $730 \%$ en el periodo más duro del confinamiento. La Hotline de la Unidad Central de Ciberdelincuencia del CNP, por su parte, registró entre el 15 de marzo y el 30 de abril 1732 avisos y/o denuncias ciudadanas por detección online de material pedófilo, lo que supuso un incremento del $352 \%$ respecto al mismo periodo de 2019 .

Se confirma, por tanto, la hipótesis de que los cambios sociales y tecnológicos, en cuanto suponen un cambio en las actividades cotidianas, producen nuevas oportunidades delictivas.

d) Por último, es preciso plantearse si la relajación de las restricciones en el segundo semestre de 2021 ha tenido alguna repercusión en las cifras de criminalidad y en qué medida. 
De enero a septiembre de 2021 la mayoría de los delitos han sufrido un incremento porcentual con respecto al mismo periodo del año 2020, excepto los robos con fuerza en establecimiento, domicilios e instalaciones que han seguido en descenso. Es llamativo, sin embargo, el notable aumento experimentado por los delitos contra la libertad e indemnidad sexual, y concretamente la agresión sexual con penetración que se ha incrementado en un 30,6\%. Según diversas instituciones las causas de tal incremento van desde el incremento de denuncias hasta una reacción post confinamiento, pero lo cierto es que la violencia machista se vuelve a situar tanto en el ámbito de la libertad sexual como en el de la violencia de género -en el que se ha incrementado tanto el número de denuncias como el de víctimas- a niveles prepandémicos.

Estos datos refuerzan la hipótesis de partida de la teoría de las actividades cotidianas, pues una vez "normalizada" la situación, hemos vuelto a nuestras actividades cotidianas y las oportunidades delictivas han vuelto a incrementarse.

Este incremento de la delincuencia en el espacio físico no ha supuesto, sin embargo, una disminución de la ciberdelincuencia. El crimen ha llegado al ciberespacio para quedarse, y a medida que nuestras actividades cotidianas se vayan trasladando al ciberespacio, se incrementará el número del total de las infracciones penales cometidas en el mismo, produciéndose un desplazamiento del crimen del espacio físico al espacio virtual.

\section{BIBLIOGRAFÍA}

BRANTINGHAM, P. J., BRANTINGHAM, P. L. (1991). Environmental criminology. Prospect Heights, IL: Waveland Press.

COHEN, L.E., FELSON, M. (1979), "Social Change and Crime Rate Trends: A Routine Activity Approach”, American Sociological Review, Vol. 44, No. 4 (Aug.), pp. 588-608.

FELSON, M., CLARKE, R. (1998), “Opportunity Makes the Thief. Practical theory for crime prevention”. Police Research Series, Paper 98. Home Office, Policing and Reducing Crime Unit, Londres.

MIRÓ LLINARES, F. (2011). "La oportunidad criminal en el ciberespacio. Aplicación y desarrollo de la teoría de las actividades cotidianas para la prevención del cibercrimen”, Revista Electrónica de Ciencia Penal y Criminología, núm. 13-07, p. 07:1-07:55. 